\title{
An Automated Approach to Detecting Signals in Electroantennogram Data
}

\author{
D. H. Slone $\cdot$ B. T. Sullivan
}

Received: 13 January 2007 /Revised: 16 May 2007 / Accepted: 20 June 2007 /

Published online: 1 August 2007

(C) Springer Science + Business Media, LLC 2007

\begin{abstract}
Coupled gas chromatography/electroantennographic detection (GC-EAD) is a widely used method for identifying insect olfactory stimulants present in mixtures of volatiles, and it can greatly accelerate the identification of insect semiochemicals. In GCEAD, voltage changes across an insect's antenna are measured while the antenna is exposed to compounds eluting from a gas chromatograph. The antenna thus serves as a selective GC detector whose output can be compared to that of a "general" GC detector, commonly a flame ionization detector. Appropriate interpretation of GC-EAD results requires that olfaction-related voltage changes in the antenna be distinguishable from background noise that arises inevitably from antennal preparations and the GC-EAD-associated hardware. In this paper, we describe and compare mathematical algorithms for discriminating olfactiongenerated signals in an EAD trace from background noise. The algorithms amplify signals by recognizing their characteristic shape and wavelength while suppressing unstructured noise. We have found these algorithms to be both powerful and highly discriminatory even when applied to noisy traces where the signals would be difficult to discriminate by eye. This new methodology removes operator bias as a factor in signal identification, can improve realized sensitivity of the EAD system, and reduces the number of runs required to confirm the identity of an olfactory stimulant.
\end{abstract}

Keywords Olfaction - Antennogram S Statistical model $\cdot$ Semiochemical ·

Signal-to-noise ratio

Electronic supplementary material The online version of this article (doi:10.1007/s10886-007-9338-6) contains supplementary material, which is available to authorized users.

D. H. Slone (区)

USGS Florida Integrated Science Center, 2201 NW 40th Ter., Gainesville, FL 32605, USA

e-mail: dslone@usgs.gov

B. T. Sullivan

USDA Forest Service, Southern Research Station, Forest Insect Research, 2500 Shreveport Hwy., Pineville, LA 71360, USA

Springer 


\section{Introduction}

Coupled gas chromatography-electroantennographic detection (GC-EAD) is a widely used procedure for distinguishing insect olfactory stimulants within mixtures of volatile chemicals. Insect antennae are exposed to compounds eluting from a gas chromatograph (GC) while the electrical potential across them is continuously recorded (Arn et al. 1975). Voltage deflections are detected in the antennae coincident with the elution of olfactory stimulants, and stimulants of particular biological importance for the insect, such as pheromones, typically produce relatively larger deflections. The elicitation and comparative strength of antennal responses can be used to assess the likelihood that a particular compound will be found (after further investigation) to have behavioral activity for an insect. GC-EAD has been used effectively by researchers to rapidly screen large numbers of candidate semiochemicals or raw extracts of active material for further evaluation in behavioral experiments, thereby eliminating unnecessary behavioral tests of inactive compounds (Cork et al. 1990; Bjostad 1998).

The utility of EAD data depends greatly upon the quality of the signal detected from the antenna and the signal-to-noise ratio. Noise can be generated within the antennal preparation and apparatus by sources of electromagnetic radiation, changing local electric fields, changes in the quality of electrode contact with the antenna, amplifier noise, as well as movement of the antennae caused by air fluctuations, muscular contractions, or mechanical vibrations conducted through the equipment (Van der Pers 1998). This constant stream of random background noise and spurious electrical perturbations invariably mask some true signals (i.e., potentials presumably arising from stimulated olfactory sensilla), and otherwise compete with them for recognition (Fig. 1). Generally, authors have reported EAD-detected olfactory stimulation without indicating a specific method for discriminating signal from noise. In some cases, authors have stipulated "consistency" or "repeatability" of an EAD spike at specific retention times as evidence for an olfaction-generated signal (Ellner et al. 2001; Nunez et al. 2002). To improve the repeatability of EAD data interpretation, some have employed explicit tests based in the statistical improbability of a random noise spike both exceeding a threshold level and recurring at the same retention time (Zhang et al. 2001; Zhang and Aldrich 2003; Asaro et al. 2004). Such methods gain power through repeated runs of the same compound over the same insect species. However,

Fig. 1 A composite EAD trace illustrating various types of deflections that may be present. The lower trace is the raw unfiltered EAD trace as it is recorded from the antenna, and the upper trace has had zero-filtering and some smoothing applied. Nonsignal "spikes" are shown at locations $a$, baseline shifts are shown at $b$, and an olfaction generated signal is shown at $c$. Smaller random noise perturbations are seen throughout

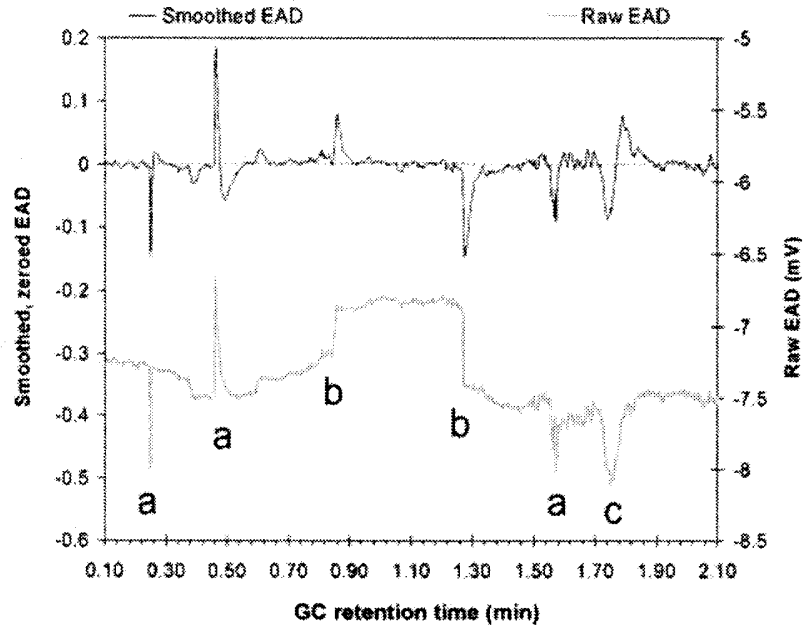

Springer 
because these methods discriminate signals using deflection amplitude data alone without considering deflection shape, they may require many data runs to resolve genuine olfactory responses, and they may be ineffective at distinguishing signals when noise-associated deflections are similar in amplitude.

The signal-to-noise ratio in EAD procedures can sometimes be improved with modifications to the associated hardware and antennal preparation technique. For example, the signal-to-noise ratio of an EAD preparation that utilizes a single antenna can be improved by attaching multiple antennae in parallel to the recording electrodes (Park and Baker 2002). In addition, the unique dynamics of olfactory responses can be exploited in the use of filters that discriminate between signal and noise. Voltage deflections produced by antennae exposed to olfactory stimulants have a characteristic shape and duration (or wavelength) that is the product of both the dose/time characteristics of the presented stimulus and antenna physiology (Sullivan and Slone, unpublished data). Consequently, low-pass filters have been incorporated into EAD amplifiers that attenuate short wavelength signals typical of electromagnetic noise while passing the long (i.e., $>1-10 \mathrm{sec}$ ) wavelengths associated with electrophysiological responses (Guerin et al. 1983; Bjostad 1998; Sasaerila et al. 2000; Leal et al. 2001). The analog EAD signal output is commonly converted to a digital stream for data storage and examination with graphical software. With the data recorded as a series of numbers representing the antennal voltage measured at a fixed interval (e.g., every $0.2 \mathrm{sec}$ ), mathematical filters can also be applied to antennogram data sets post-collection to extract antennal response from background noise. Such post hoc methods are available on commercial EAD software and include smoothing algorithms such as moving-average which, like low-pass filters, tend to attenuate noise with shorter wavelengths. However, the shape dynamics of olfaction-generated EAD signals are adequately distinct to allow discrimination based on more precise waveform characteristics; hence, further refinement to such post hoc noise filters is possible. Assuming the concentration of stimulant eluting from the GC is not approaching the saturation point of the olfactory sensillae, EAD output amplitude will covary approximately linearly with the log-transformed concentration of an eluting stimulant (Zhang et al. 2002; Sullivan, unpublished data) or the first integral of the amplitude of the flame ionization detector output. Hence, the shape of the raw olfaction-generated signal closely resembles that of the flame ionization detector (FID) peak. Under normal GC operating conditions (i.e., the column is not overloaded and its temperature is programmed to increase constantly), these peaks should resemble the bell shape of a normal distribution (Fig. 2). Hence, EAD peaks arising from GC-generated stimuli eluting will have an essentially constant wavelength

Fig. 2 A GC peak and associated EAD olfaction response superimposed to show the approximate bell shape of each

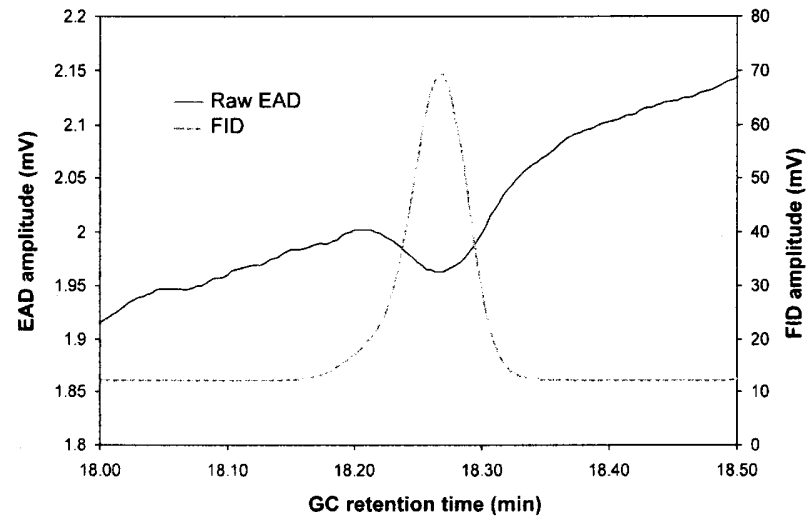


(i.e., peak width) and a high degree of bilateral symmetry between their left and right slopes. In this paper, we describe three algorithms that discriminate olfaction-generated signals from noise in EAD data on the basis of either (1) signal amplitude alone, (2) signal amplitude and wavelength, or (3) signal amplitude, wavelength, and symmetry. We then compare the signal-discriminating capacity of the three algorithms to one another and to discrimination methods based on either general visual appearance of EAD traces or retention time consistency of signals. The algorithms are formulated to be applicable to any EAD data sets where voltage changes across antennae are detected with a high-impedance DC amplifier (such as described in Bjostad 1998 and used in EAD systems sold by Syntech, Hilversum, The Netherlands) interfaced with an analog-to-digital converter and data management software that allows the final collected data set to be converted to an ASCII file and examined in a spreadsheet program. PC-based digital data acquisition systems suitable for GC-EAD commonly permit the user to convert recorded data to ASCII format (including data acquisition systems sold by Syntech; SRI Instruments, Torrance, CA, USA; and DATAQ, Akron, OH, USA), so it should be possible for most GC-EAD operators to apply our algorithms to their own data sets. In addition, we have made available on the Internet an Excel spreadsheet that performs all of the calculations.

\section{Methods and Materials}

Apparatus Setup and Data Collection EAD data were collected from the antennae of the Nantucket pine tip moth, Rhyacionia frustrana (Comstock; Lepidoptera: Tortricidae), the southern pine beetle, Dendroctonus frontalis Zimmermann (Coleoptera: Curculionidae), and the parasitoid Roptrocerus xylophagorum (Ratzeburg; Hymenoptera: Pteromalidae) using procedures and apparatus described previously (Pettersson et al. 2000; Asaro et al. 2004; Sullivan 2005). Antennae were mounted between two glass electrodes filled with Beadle-Ephrussi ringer solution amended with polyvinylpyrrolidone (Bjostad 1998), and the recording electrode was connected to a high impedance guarded input AC/DC preamplifier (Syntech). Output from the preamplifier was processed by a signal interface box (Syntech IDAC 2/3) that provided optional baseline control of the analog signal. Digitized signal from the FID and the signal interface box were collected at five samples per second with a PeakSimple Chromatographic Interface and PeakSimple software (SRI instruments). Data were collected without the automatic baseline return filter engaged on the IDAC 2/3. The EAD and FID data files were saved in ASCII format and subsequently opened in an Excel spreadsheet as two columns of successive voltage readings from the FID and the EAD [note that for data collected with Electro Antenno Detection software (Syntech 2004), "EAD session" data files (*.ead) can be saved in ASCII format (*.asc) within the "File: Save As" window].

Data Transformation We first applied an exponential zeroing filter to the raw EAD data. Filtrations of the data in this way converted the approximately Gaussian raw EAD signals to "sigmoid" deflections that were essentially identical to the output produced by the analog baseline-return filter present on the SYNTECH IDAC 2/3 signal interface box:

$$
Z_{t}=E_{t}-E_{t-1}+r \cdot Z_{t-1}
$$

where $Z=$ zeroed data, $t=$ sample number, $E=\mathrm{EAD}$ data (i.e., voltage recorded from antenna), and $r=$ zeroing rate [related to the time constant (T.C.) on the SYNTECH IDAC 2/ 3 by $r=0.37^{\frac{1}{s_{s} \mathrm{TC}}} ; f_{\mathrm{s}}=$ sampling rate per second. We set $r$ to a value of $0.8 w^{0.05}$ where $w$ is 
the width in samples of an average FID peak in the sample run at half its height. This value produced positive and negative deflections in the zeroed EAD data that were similar in amplitude.

We further filtered the zeroed data to remove spurious noise spikes by applying an exponential averager (Lyons 1997):

$$
s_{t}=\alpha_{s} \cdot Z_{t}+\left(1-\alpha_{s}\right) \cdot s_{t-1},
$$

where $\alpha_{s}=$ weighting factor for smoothing. The purpose of the averaging filter is to pass deflections that occur at the wavelength expected of an olfaction signal (for EAD data, this is the wavelength of the FID peaks) while attenuating the higher frequencies associated with noise. For typical GC-EAD recordings where the FID peak width at half-height is $2-3 \sec (10$ 15 time steps), we found that a value of 0.15 for $\alpha_{\mathrm{s}}$ maximized the difference in amplitude between signal peaks and higher frequency noise, thus allowing for the highest $\mathrm{S} / \mathrm{N}$ ratio.

We calculated a baseline using a similar exponential averaging function:

$$
b_{t}=\alpha_{\mathrm{b}} \cdot s_{t}+\left(1-\alpha_{b}\right) \cdot b_{t-1}
$$

where $\alpha_{\mathrm{b}}=$ weighting factor for the baseline. The baseline is the axis from which we measured height of EAD deflections. Hence, it ideally should attenuate signals with the wavelength expected from olfaction signals while passing lower frequencies, thereby allowing the baseline to follow the average meanderings of the zeroed EAD data and permitting the subtraction of baseline "drift". To achieve this end, we set $\alpha_{b}$ to 0.001 .

Signal Discrimination We transformed the data finally with one of three candidate algorithms designed to "filter out" EAD deflections that did not possess specific distinguishing characteristics of those produced by olfactory stimulants eluting from the GC. Our goal was to design a function whose value $(\psi)$ was large and positive when an olfaction-generated signal occurred at retention time $t$, and small or negative otherwise.

The first algorithm was the Peak Height method. The amplitude of negative EAD deflections from the baseline was measured at time $t$. This algorithm formalized the process ostensibly used by EAD operators during visual evaluation of graphic EAD output, namely, locating negative deflections in zeroed data that stand out appreciably from the background noise:

$$
\psi_{P(t)}=b_{t}-s_{t},
$$

The second algorithm was the Additive method. In addition to the initial downward deflection of the EAD output, a second positive deflection is generated by the zeroing filter (Eq. 1) when the raw data stream contains a negative-deflecting Gaussian peak of a wavelength similar to the GC peaks. The amplitude of this positive deflection will equal approximately that of the negative deflection when the EAD response possesses the same wavelength (i.e., peak-width) as a GC-generated stimulus (i.e., an FID peak), when $r$ of the zeroing algorithm is set to $0.8 w^{0.05}$. Hence, for EAD signals generated by GC-eluting stimuli, that is, "true" olfactory responses, the sum of the amplitudes of a negative deflection occurring at time $t$ and a corresponding positive deflection occurring at time $t+w$ will have a value that exceeds that produced by random background noise comprised of random frequencies:

$$
\psi_{A(t)}=\left(b_{t}-s_{t}\right)+\left(s_{t+w}-b_{t+w}\right) .
$$

This algorithm tends to filter out EAD deflections with either low amplitude and/or inappropriate wavelength, and therefore, was hypothesized to be more accurate at Springer 
discriminating signal from noise than the "Peak Height" algorithm (Eq. 4) which discriminated signals by using amplitude alone.

Finally, in the Minimum method, we recorded the smallest of the negative and positive deflections of the zero-filtered EAD data stream at the wavelength expected from an olfaction-generated peak as the value of the function:

$$
\psi_{M(t)}=\min \left[\left(b_{t}-s_{t}\right),\left(s_{t+w}-b_{t+w}\right)\right] .
$$

This method discriminated specifically against EAD deflections in the zeroed data where the negative deflection at time $t$ and positive deflection at $t+w$ differed substantially from each other in amplitude: higher function values are generated for peaks with both the symmetry of a Gaussian peak and appropriate wavelength. Because this algorithm discriminated peaks based on three diagnostic qualities of olfaction-generated EAD signals (i.e., peak amplitude, wavelength, and symmetry), we hypothesized that this algorithm might result in greater rejection of noise than the two previously described.

Noise Characterization In the absence of compounds eluting from the GC, EAD output had a continuous and predictable noise pattern. The distribution of noise around the baseline was normally distributed. In addition, we found that the individual points had a high degree of positive serial correlation, with first degree correlations typically above 0.8 (Slone and Sullivan, unpublished data). Serial correlation in the data peaks would invalidate traditional statistical techniques by violating the assumption of independence.

Signal and Noise Measurement We defined a "peak" as any location where the following was true:

$$
\psi_{t}=\max \left(\psi_{t-2}, \psi_{t-1}, \psi_{t}, \psi_{t+1}, \psi_{t+2}\right) .
$$

By using only the peaks, we reduced the amount of points to process and eliminated the $\sim 0.8$ first-order serial correlation present in EAD data (Slone and Sullivan, unpublished data). These peak values were normally distributed around their mean, which allowed us to use conventional statistical techniques.

To categorize each peak as either signal or noise, we first identified sections of the processed EAD run $(\psi)$ where elution of olfactory stimuli from the GC could not have occurred. These sections included retention times both before the solvent peak eluted from the column (but after the initial antenna noise had abated) and after all analytes had eluted at the end of the GC temperature program. We then calculated the mean $\left(\mu_{n}\right)$ and standard deviation $(\sigma)$ of all positive peaks within those sections as an estimate of baseline noise inherent in the entire EAD trace.

Next, a cutoff amplitude was selected to separate true signal peaks above the cutoff from noise peaks below the cutoff. This amplitude was set through a combination of the noise distribution and the desired probability of committing a type I (false identification of noise as signal) error. The experimentwise error probability ( $\alpha_{\mathrm{E}}$; typically 0.05$)$ depended upon the number of peaks in the data set and the probability that any single peak might trigger a type I error (comparisonwise error, $\alpha_{\mathrm{c}}$ ). The $\alpha_{\mathrm{E}}$ and $\alpha_{\mathrm{c}}$ values are related by the following equation, functionally similar to the Bonferroni correction (Jones 1984):

$$
\alpha_{c}=1-\left(1-\alpha_{E}\right)^{1 / n},
$$

where $n$ is the total number of peaks in the portion of the data stream that is being tested. The cutoff amplitude was calculated by finding the value of the $z$ statistic that satisfied the 
following equation of the normal distribution populated with the calculated values of $\alpha_{\mathrm{c}}$ and $\sigma$. (e.g., Mendenhall et al. 1990):

$$
\alpha_{c}=\int_{y=z}^{\infty} \frac{1}{\sigma \sqrt{2 \pi}} \exp \left[-\left(\frac{1}{2 \sigma^{2}}\right) y^{2}\right]
$$

and adding that value to the mean noise level $\left(\mu_{\mathrm{n}}\right)$. Any peak that had a value greater than $\left(\mu_{\mathrm{n}}+z\right)$ was categorized as a signal, and all others were rejected as noise.

Performance Testing: Simulation We evaluated the sensitivity and noise-rejection capacity of the algorithms by applying them to simulated EAD data sets that contained a specified amplitude of random background noise and either a simulated olfaction signal (a normally distributed deflection of known amplitude and a width at half-height of $w$ ), a spurious, short-duration deflection event (spike), also of known amplitude, or an instantaneous baseline-shift of known amplitude (see Fig. 1). For each simulation, $t_{\max }=3,000$, with 1,000 samples containing only background noise (used for measuring $\mu$ and $\sigma$ of the noise) and 2,000 samples containing background noise plus the simulated signal/spurious event, and each simulation was replicated 20 times The specific tests we performed were as follows:

1. Twenty well-spaced simulated olfaction signals with background noise repeated at several signal/noise ratios

2. Positive and negative simulated spurious spikes randomly generated at a probability of 0.0125 per time step ( $\sim 25$ spikes per run; these spikes were randomly generated to allow for any effects from varied spacing and positive-negative spike interactions). These runs were also repeated with the spikes occurring over a range of amplitudes.

3. Instantaneous positive and negative baseline shifts, randomly generated at a probability of $0.0125(\sim 25$ shifts per run; the shifts were randomly generated for reasons described in 2 above).

Performance Testing: Real Data We applied the algorithms to real data sets and compared their performance. First, we examined EAD runs from $R$. frustrana, D. frontalis, and $R$. xylophagorum and then compared FID and EAD traces at retention times where the different algorithms indicated antennal signals.

Second, we applied the algorithms to noisy EAD runs of $D$. frontalis females exposed to analyzed extracts of hindguts of $D$. frontalis males. Eleven electrophysiologically active molecules had been previously identified for this combination of insect and test extract (Sullivan 2005). We processed 17 individual EAD runs using experimental error rates $\left(\alpha_{E}\right)$ from a conservative $2.7 \mathrm{e}-6$ to a liberal 0.9999 . For each run, we calculated the baseline noise level and cutoff amplitude using EAD output after the solvent peak, but before the first FID-detectable sample peak eluted from the GC, and again after no further peaks were detected by the FID. We noted the number of signals detected by each algorithm that matched the retention times of the 11 known active molecules and also any signals detected at other retention times. Similarly, we performed 17 replicates with each algorithm where the digital EAD output from two runs selected at random from the $17 \mathrm{D}$. frontalis/hindgut runs were averaged, then 17 replicates of four runs averaged, 17 replicates of eight runs averaged, and finally one replicate of all 17 runs averaged. These runs were all processed with experimental error rates $\left(\alpha_{\mathrm{E}}\right)$ of 0.05 . This method of averaging multiple EAD runs 
from different antennae to improve the signal-to-noise ratio was previously used by Sullivan (2005).

\section{Results and Discussion}

Simulation Testing Tests of the three algorithms that used simulated EAD runs revealed the strengths and weaknesses of each, with highly significant performance differences $(P<<0.05)$ found among the algorithms in all tests. The Peak Height method was always inferior in finding signals to the other two methods, and the Additive method was significantly better than the Minimum method (Fig. 3). In terms of noise rejection, the Peak Height method was also inferior to the other two methods in cases where the spike amplitude was less than eight times, or the baseline shift amplitude was less than three times, the maximum background noise amplitude (99th percentile of all noise peaks). With very large spikes or baseline shifts, the Additive method rejected less noise than the other methods. The superior method for noise rejection was clearly the Minimum method, which rejected large spikes and baseline shifts five to ten times better than the other methods.

The simulation testing was deliberately designed to highlight marginal cases where olfaction-generated signals were competing with large amounts of background noise. For EAD runs where the amplitude of olfaction-produced deflections stands well beyond the background (i.e., those performed with especially good antennal preparations and morethan-adequate concentrations of stimulants in the test sample), most signals would be easily discerned by any of the algorithms or by eye. However, there are often cases where the amplitudes of some or all of the signals are small, or the number of spurious deflection events in the run are large, and in these situations, the Additive or Minimum methods should provide EAD operators with a powerful tool for improving their ability to discriminate noise from signal. If the operator's goal is to identify all possible signals in a run with the possibility of encountering some false positives, then the Additive method is preferable. If the goal is to minimize errors, then the Minimum method is superior. The discriminatory power of the algorithms can be enhanced several-fold if applied to data from

Fig. 3 The proportion of simulated signals that each of the algorithms discriminated is shown as the mean of 20 replications plus standard deviation error bars at each of several signal to noise ratios. An arcsin (sqrt) transformation was used to linearize the proportional results. For $\mathrm{S} / \mathrm{N}$ ratios $<1.5$, the Additive method was slightly superior to the Minimum method, and both were far better at discriminating the simulated signals than the Peak Height method. For $\mathrm{S} / \mathrm{N}$ ratios $\geq 1.5$, all methods discriminated all signals

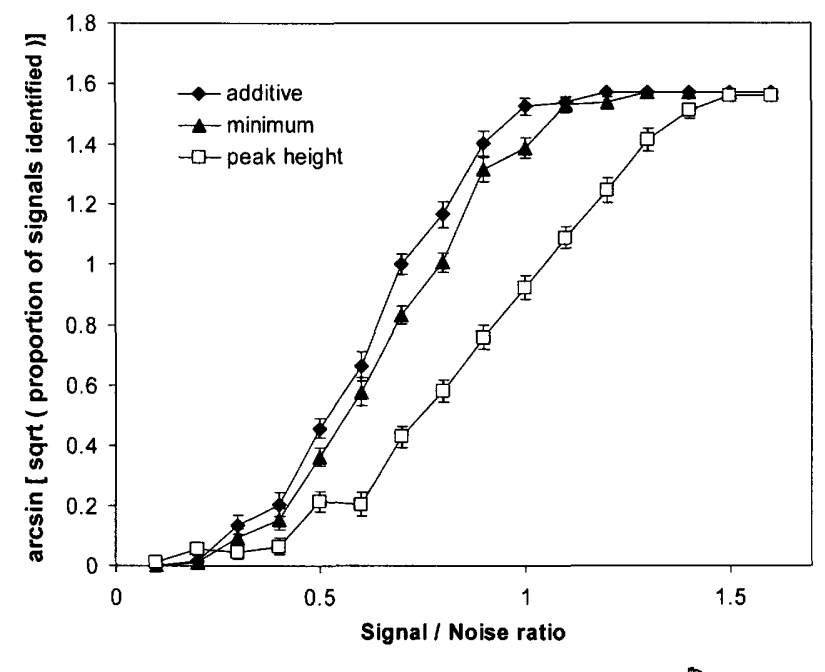




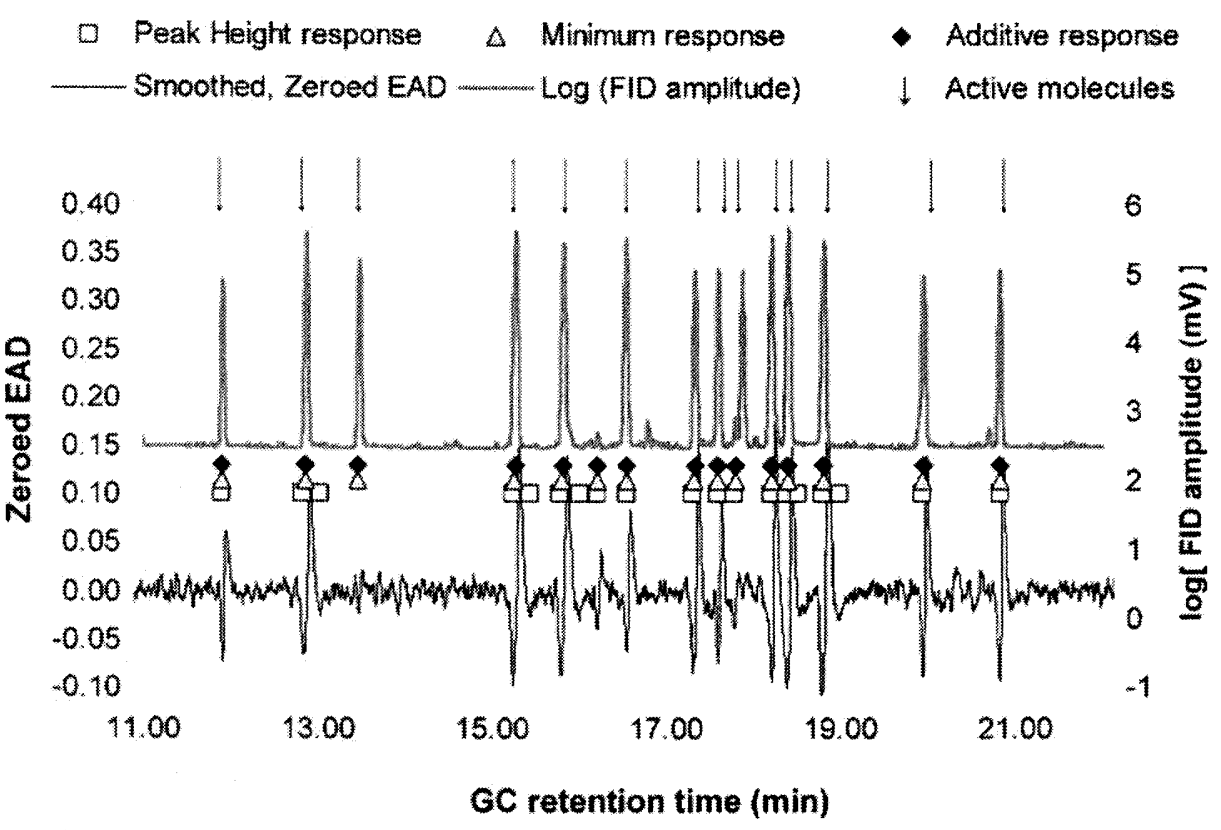

Fig. 4 Zeroed EAD recording of the parasitic wasp Roptrocerus xylophagorum (Hymenoptera: Pteromalidae) and the associated GC trace from a mix of host and associated plant odors. The locations of previously determined active molecules (arrow) and significant responses (Additive, diamond; Minimum, triangle; Peak Height, square; $\alpha_{\mathrm{E}}=0.05$ ) from the three algorithms are shown

multiple GC-EAD runs (replications) because the likelihood that a "false" signal will be registered at the same retention time will become vanishingly small with additional replication. Statistical tests can also be performed on the frequency that signals are detected at a particular retention time to further reject spurious signals and accept only the retention times of active compounds; signals should occur with a greater frequency than predicted by random chance at the retention times of active molecules (Zhang et al. 2001; Zhang and Aldrich 2003; Asaro et al. 2004). The chief contribution of these two algorithms to interpretation of EAD runs is the increase of the effective signal/noise ratio in the data, an improvement which reduces the number of EAD runs necessary for a researcher to accurately discern electrophysiologically active compounds.

Real Data Testing All three algorithms successfully discriminated antennal signals from noise in EAD data from the three orders of insects examined. In an EAD recording of the parasitic wasp $R$. xylophagorum responding to a mix of host and associated plant odors, the Additive and Minimum methods correctly "tagged" all of the active compounds [i.e., compounds whose activity with olfactory sensillae had been demonstrated by statistical analysis of multiple EAD runs (Pettersson et al. 2000; Sullivan, unpublished) in the mixture (Fig. 4)]. The Peak Height method failed to detect one active compound and returned five apparent false positives at the tail end of active compounds. All methods identified a compound at 16.21 min that has not previously been demonstrated as an active molecule. In a rather noisy EAD recording of the southern pine beetle, $D$. frontalis, subjected to the same mix of odors as $R$. xylophagorum above (Sullivan, unpublished), all three methods identified signals at all three retention times of compounds with demonstrated olfactory and/or behavioral activity with this beetle (Fig. 5). Additionally, all three methods identified 


$\begin{array}{cc}\text { Peak Height response } \triangle \text { Minimum response } & \bullet \text { Additive response } \\ \text { Smoothed, Zeroed EAD } & \downarrow \text { Active molecules }\end{array}$

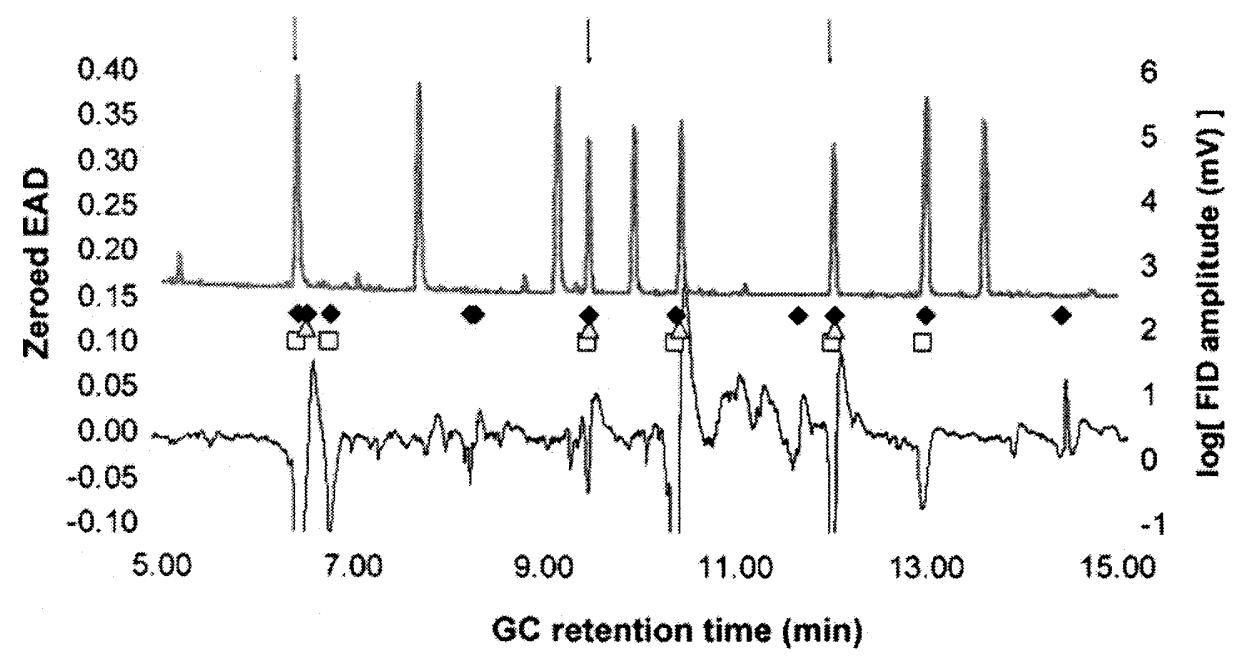

Fig. 5 Zeroed EAD recording of the southern pine beetle, Dendroctonus frontalis (Coleoptera: Curculionidae) subjected to the same mix of odors as $R$. xylophagorum in Fig. 6 . The locations of previously determined active molecules (arrow) and significant responses (Additive, diamond; Minimum, triangle; Peak Height, square; $\alpha_{\mathrm{E}}=0.05$ ) from the three algorithms are shown

a rather large peak at approximately 10.50 min associated with the elution of $p$-cymene, although this has not previously been demonstrated to be an olfactory stimulant. While the Minimum method returned no additional positives, the Additive method detected seven additional active retention times that were apparently false positives, and the Peak Height method returned two additional false positives. In an EAD recording of a female Nantucket pine tip moth ( $R$. frustrana) exposed to a mixture of host-associated odors (Asaro et al. 2004), all three algorithms correctly identified signals at retention times of seven known olfactory stimulants in the mixture (Fig. 6). The Additive method also detected one additional active molecule at 15.70 min that has not been identified previously as an olfactory stimulant for this insect, and it returned an additional two false positives. The Peak Height and Minimum methods failed to detect two known active molecules, and the Peak Height method returned a single false positive. All algorithms also identified one FID peak at approximately $16.50 \mathrm{~min}$ that had not been shown previously to be an active compound.

The replicated EAD runs from $D$. frontalis females that responded to male $D$. frontalis hindgut extract were relatively noisy. In general, approximately half of the retention times associated with electrophysiologically active compounds were identified at $\alpha_{\mathrm{E}}=0.05$, but with clear differences in performance among the algorithms (Fig. 7). The Additive method consistently identified more "true" signals than the other two algorithms at any $\alpha_{\mathrm{E}}$ level, yet tagged fewer erroneous retention times than the Peak Height method. The Minimum method identified fewer signals than the other methods, but showed greater error-rejecting capability by not tagging any retention times incorrectly at $\alpha_{\mathrm{E}}$ levels $\leq 0.05$.

Although the Minimum method had superior error-rejection capacity, the Additive method showed the best overall power by consistently producing the fewest false 
a Peak Height response $\triangle$ Minimum response

Smoothed, Zeroed EAD $-\log ($ FID amplitude)
- Additive response

1 Active molecules

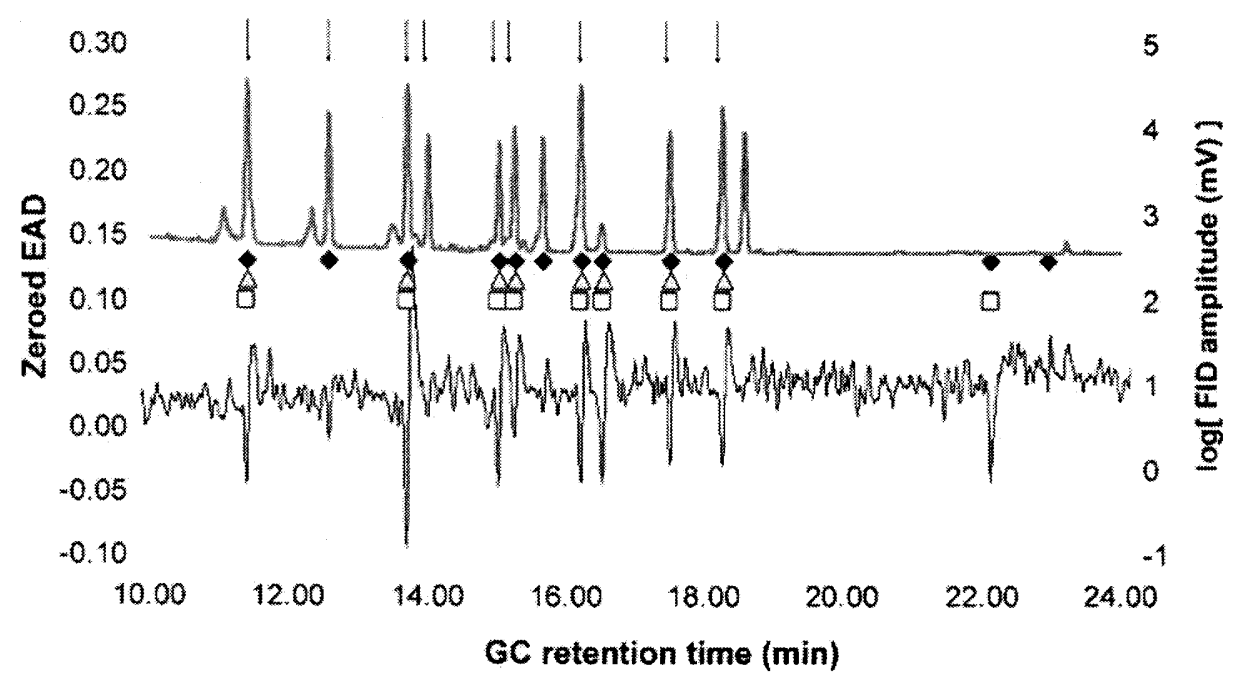

Fig. 6 Zeroed EAD recording of a female Nantucket pine tip moth, Rhyacionia frustrana (Lepidoptera: Tortricidae) and the associated GC trace from a mixture of host-associated odors. The locations of previously determined active molecules (arrow) and significant responses (Additive, diamond; Minimum, triangle; Peak Height, square; $\alpha_{\mathrm{E}}=0.05$ ) from the three algorithms are shown

classifications per correct classification (Fig. 8). The Minimum method had medium overall power, and the Peak Height method had the lowest power with real data sets, returning the highest number of false positives per true response. Algorithm traces for the Additive method also consistently had higher average signal/noise ratios than output from the other algorithms (Fig. 9). Additionally, the Additive method recognized more true olfactiongenerated signals than is apparently possible by visually comparing peak heights in EAD

Fig. 7 Average number of true and false significant responses $( \pm \mathrm{l}$ SD) from 17 southern pine beetle EAD runs produced by each algorithm using a range of experimental error rates $\left(\alpha_{\mathrm{E}}\right)$. The numbers of signals found were $\log (x+0.1)$-transformed before averaging to normalize the variances

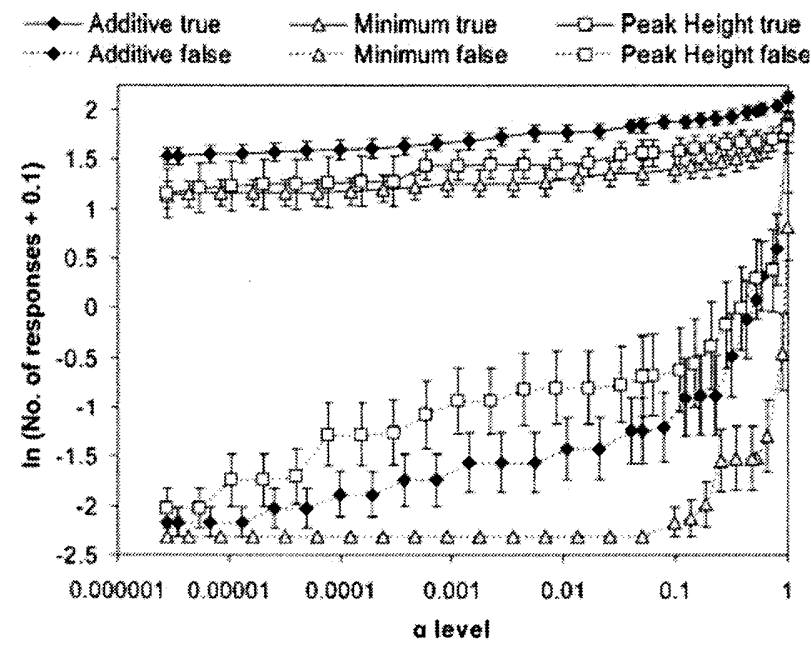


Fig. 8 True vs. false significant responses returned from each algorithm for southern pine beetle EAD runs. Each point shown represents the average of 17 runs evaluated at a different $\alpha_{\mathrm{E}}$ level, ranging from $2.7 \mathrm{e}-6$ (farthest left) to 0.9999 (farthest right). Points lower and further to the right indicate superior performance

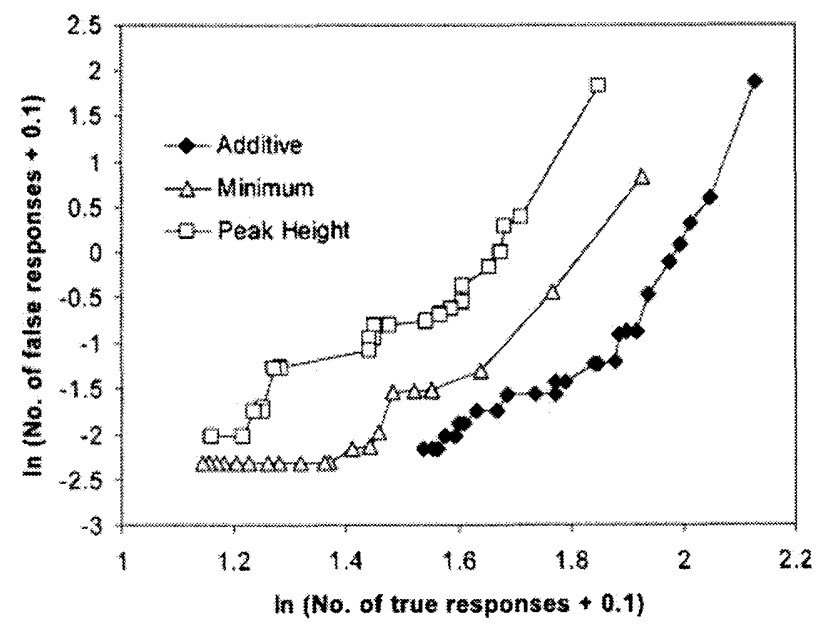

traces, as this latter procedure was ostensibly duplicated by the Peak Height algorithm. Thus, our data demonstrate that the Additive algorithm was an effective "amplifier" of olfaction-generated responses in insect-produced EAD traces.

Our analyses of averaged data from randomly selected combinations of EAD replicates showed that greater signal-resolving power and improved noise rejection occurred when the algorithms were applied to averaged data from progressively greater numbers of runs

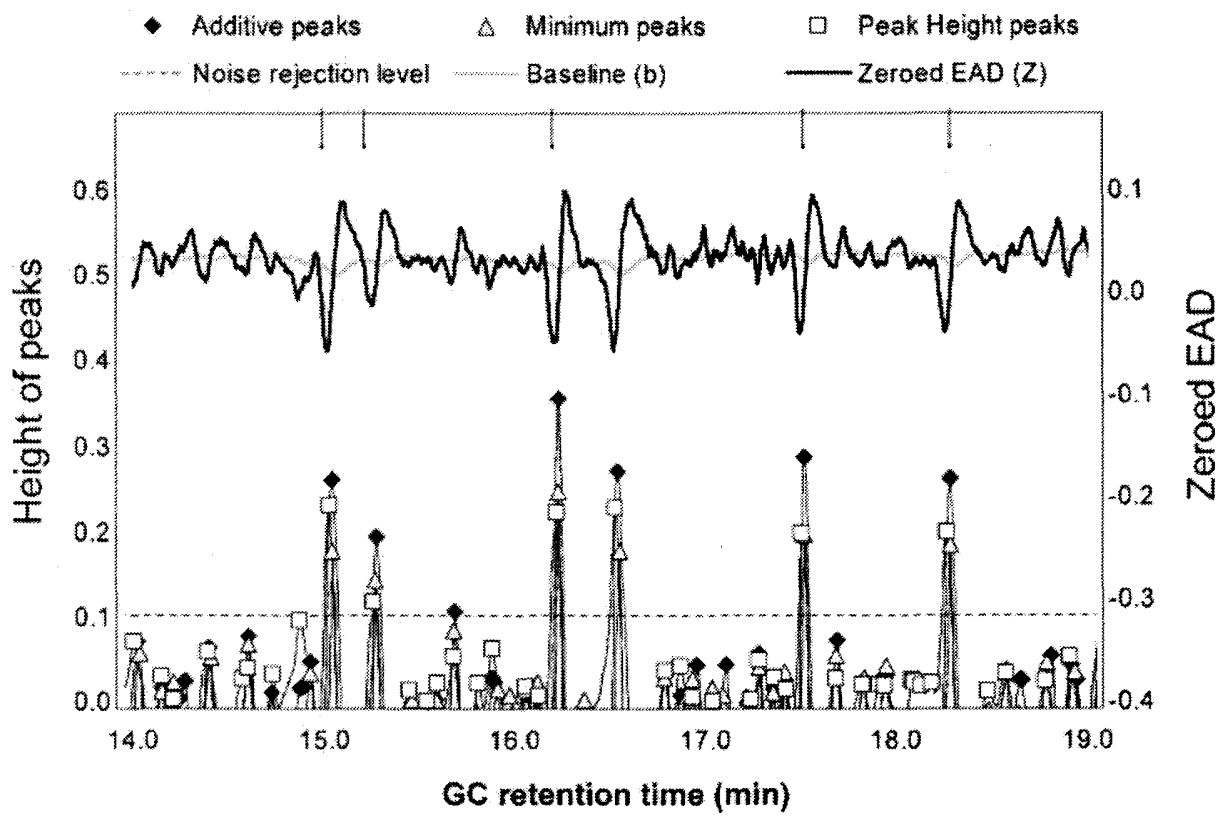

Fig. 9 Output traces $(\psi)$ from each of the algorithms are shown below a section of EAD output from Fig. 6 , a female Nantucket pine tip moth, Rhyacionia frustrana (Lepidoptera: Tortricidae) responding to a mix of host-associated odors. The output from each method has been standardized so that the mean of the trace is 0 , and the noise rejection limit is 0.1 . The Additive method amplified EAD olfaction peaks relative to noise peaks more than the Minimum or Peak Height methods 
Fig. 10 Results from southern pine beetle EAD run averaging showing increasing numbers of true responses and decreasing numbers of false responses when more runs were averaged. With all 17 runs averaged, the Additive method found all 11 active molecules with no errors. Data were $\log (x+0.1)$-transformed for analysis and back-transformed for display. Note that the true and false responses are displayed on different scales for clarity

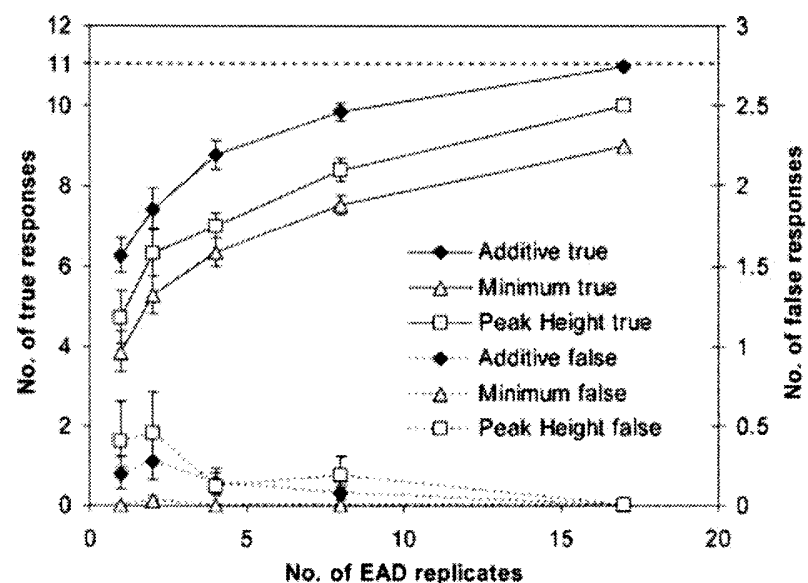

(Fig. 10). With $\alpha_{\mathrm{E}}=0.05$, the Additive method consistently identified the greatest number of retention times associated with active compounds while committing a modest number of errors, and it was the only method to tag the retention times of all 11 active molecules when all 17 runs were averaged. When four or more runs were averaged, the Additive method also consistently identified a location at $22.75 \mathrm{~min}$ corresponding to an unknown singly oxygenated monoterpene. Because this was an unknown compound with uncertain biological activity, it was excluded from the analysis and was not counted as either correct or incorrect. The Peak Height method identified a moderate number of active retention times, but it also had the highest number of apparent false positives. The Minimum method identified the fewest signals, but it generally had no false positives. Because the EAD traces of southern pine beetle responding to hindgut extracts were generally noisy, several replicated analyses of averaged run sets were necessary to resolve all 11 active molecules. Nonetheless, the analysis in Fig. 10 demonstrates that both the Minimum algorithm and, to a greater extent, the Additive algorithm can reduce the number of EAD runs necessary for discerning electrophysiologically active compounds.

Applications Our Additive algorithm represents a powerful tool that can be used to enhance the realized signal-to-noise ratio of any GC-EAD system that stores its data digitally. Raw data can be copied into a spreadsheet program, transformed, and then examined using the graphics capabilities of the spreadsheet. We created an Excel 2000 spreadsheet that contains all of the required transformations and calculations. The user pastes raw or pre-zeroed EAD data into a column, selects areas of the data with no eluting molecules to calibrate the noise level, and enters other information about the data collection methodology. Charts on the spreadsheet display the traces and indicate significant impulses found by each of the three methods. This spreadsheet is available as electronic supplementary material (doi:10.1007/ s10886-007-9338-6).

In summary, with a novel insect or compound, the optimal use of GC-EAD data for identifying olfactory stimulants is of great importance, as an incorrect identification of a stimulant could lead a researcher seeking behavior-modifying compounds to waste effort on an inactive compound; in contrast, failure to recognize evidence of olfactory stimulation could cause an important behavioral molecule to be overlooked. For antennal preparations of some insect species, the maximum signal level obtainable may be at or below the 
background noise level (for example, see Russell et al. 2004). As our Additive and Minimum method algorithms have the capacity for extracting signals that are similar in amplitude to surrounding noise, they should effectively increase the sensitivity of an EAD system and may allow data collection where it would not otherwise have been possible. Additionally, our algorithms and data analysis procedures eliminate the potential for experimenter bias that is inherent in visual evaluation of data sets because the decision of whether a deflection event is a "real" signal or not is made by a uniformly applied mathematical test. By combining these algorithms with replication, high levels of power and discrimination for detecting active molecules in EAD data are provided.

Acknowledgments The bulk of this work was completed at the USDA Forest Service Forest Insect Research facility while the first author was employed there. The authors thank two anonymous reviewers for their thoughtful comments.

\section{References}

ARN, H., STÄDLER, E., and RAUSCHER, S. 1975. The electroantennographic detector--A selective and sensitive tool in the gas chromatographic analysis of insect pheromones. Z. Naturforsch 30c:722-725.

Asaro, C., Sullivan, B. T., Dalusky, M. J., and Berisford, C. W. 2004. Volatiles associated with preferred and nonpreferred hosts of the Nantucket pine tip moth, Rhyacionia frustrana. J. Chem. Ecol. 30(5):977-990.

BJOSTAD, L. B. 1998. Electrophysiological methods, pp. 339-375, in J. G. Millar, K. F. Haynes, (eds.). Methods in Chemical Ecology, volume 1: Chemical Methods. Kluwer, Boston, MA.

Cork, A., Beevor, P. S., Gough, A. J. E., and Hall, D. R. 1990. Gas chromatography linked to electroantennography: A versatile technique for identifying insect semiochemicals, pp. 271-274, in A. R. McCaffery, I. D. Wilson, (eds.). Chromatography and Isolation of Insect Hormones and Pheromones. Plenum, New York.

Ellner, S. P., Mccauley, E., Kendall, B. E., Briggs, C. J., Hosseini, P. R., Wood, S. N., Janssen, A., Sabelis, M. W., TURChin, P., Nisbet, R. M., and Murdoch, W. W. 2001. Habitat structure and population persistence in an experimental community. Nature 412:538-543.

GUERIN, P. M., STÄDLER, E., and BUSER, H. R. 1983. Identification of host plant attractants for the carrot fly, Psila Rosae. J. Chem. Ecol. 9:843-861.

JONES, D. 1984. Use, misuse, and role of multiple-comparison procedures in ecological and agricultural entomology. Environ. Entomol. 13:635-649.

Leal, W. S., Bento, J. M. S., Murata, Y., Ono, M., Parra, J. R. P., and Vilela, E. F. 2001. Identification, synthesis, and field evaluation of the sex pheromone of the citrus fruit borer Ecdytolopha aurantiana. $J$. Chem. Ecol. 27(10):2041-2051.

LYONS, R. G. 1997. Understanding Digital Signal Processing. Addison Wesley Longman, Reading, MA.

Mendenhall, W., Wackerly, D. D., and Scheaffer, R. L. 1990. Mathematical Statistics with Applications. Duxbury Press, Belmont, CA.

Nunez, S., Devlieger, J. J., Rodriquez, J. J., Persoons, C. J., and SCAToni, I. 2002. Sex pheromone of South American tortricid moth Argryotaenia sphaleropa. J. Chem. Ecol. 28:425-432.

PARK, K. C. and BAKER, T. C. 2002. Improvement of signal-to-noise ratio in electroantennogram responses using multiple insect antennae. J. Insect Physiol. 48:1139-1145.

Pettersson, E. M., Sullivan, B. T., Anderson, P., Berisford, C. W., and Birgersson, G. 2000. Odor perception in the bark beetle parasitoid Roptrocerus xylophagorum exposed to host associated volatiles. J. Chem. Ecol. 26:2507-2525.

Russell, G. B., FAUnDeZ, E. H., and Niemeyer, H. M. 2004. Selection of Nothofagus host trees by the aphids Neuquenaphis staryi and Neuquenaphis edwardsi. J. Chem. Ecol. 30:2231-2241.

Sasaerila, Y., Gries, R., Gries, G., Khaskin, G., King, S., and Boo, T. C. 2000. Decadienoates: Sex pheromone components of nettle caterpillars Darna trima and D. bradleyi. J. Chem. Ecol. 26:1969-1981.

SULLIVAN, B. T. 2005. Electrophysiological and behavioral responses of Dendroctonus frontalis (Coleoptera: Curculionidae) to volatiles isolated from conspecifics. J. Econ. Entomol. 98:2067-2078.

SYNTECH Inc. 2004. Electro Antenno Detection V2.6, SYNTECH, Hilversum, The Netherlands.

VAN DER PERS, J. N. C. 1998. Electroantennography: A Practical Introduction. Syntech Inc., Hilversum, The Netherlands. 
ZHANG, Q. H., and ALDRICH, J. F. 2003. Pheromones of miltweed bugs (Heteroptera: Lygaeidae) attract wayward plant bugs: Phytocoris mirid sex pheromone. J. Chem. Ecol. 29:1835-1851.

Zhang, Q. H., LiU, G. T., SChlyter, F., BiRgersson, G., ANDERSON, P., and VAlfeur, P. 2001. Olfactory responses of Ips duplicatus from Inner Mongolia, China to nonhost leaf and bark volatiles. J. Chem. Ecol. 27:995-1009.

ZhaNG, Q. H., TOLASCH, T., SCHLYTER, F., and FrANCKE, W., 2002. Enantiospecific antennal response of bark beetles to spiroacetal (E)-conophthorin. J. Chem. Ecol. 28:1839-1852. 